\title{
Pyrosequencing reveals transient cystic fibrosis lung microbiome changes with intravenous antibiotics
}

\author{
Daniel J. Smith 1,2,3,4,8, Alison C. Badrick ${ }^{2,3,8}$, Martha Zakrzewski ${ }^{5}$, Lutz Krause ${ }^{5}$, \\ Scott C. Bell $1,4,6$, Gregory J. Anderson ${ }^{2,4}$ and David W. Reid ${ }^{1,2,3,4,7}$
}

Affiliations: 'Dept of Thoracic Medicine, The Prince Charles Hospital, Brisbane, QLD, Australia. ${ }^{2}$ Iron Metabolism Laboratory, QIMR Berghofer Medical Research Institute, Brisbane, QLD, Australia. ${ }^{3}$ Lung, Inflammation and Infection Laboratory, QIMR Berghofer Medical Research Institute, Brisbane, QLD, Australia. ${ }^{4}$ School of Medicine, The University of Queensland, Brisbane, QLD, Australia. ${ }^{5}$ Bioinformatics Laboratory, QIMR Berghofer Medical Research Institute, Brisbane, QLD, Australia. ${ }^{6}$ Queensland Children's Medical Research Institute, Brisbane, QLD, Australia. ${ }^{7}$ Menzies Research Institute, Royal Hobart Hospital, Hobart, TAS, Australia. ${ }^{8}$ Both authors contributed equally.

Correspondence: Daniel J. Smith, Dept of Thoracic Medicine, The Prince Charles Hospital, Rode Rd, Chermside QLD 4032, Australia. E-mail: daniel_j_smithahealth.qld.gov.au

ABSTRACT Chronic airway infection in adults with cystic fibrosis (CF) is polymicrobial and the impact of intravenous antibiotics on the bacterial community composition is poorly understood. We employed culture-independent molecular techniques to explore the early effects of i.v. antibiotics on the CF airway microbiome.

DNA was extracted from sputum samples collected from adult subjects with CF at three time-points (before starting treatment, and at day 3 and day 8-10 of i.v. antibiotics) during treatment of an infective pulmonary exacerbation. Microbial community profiles were derived through analysis of bacterial-derived $16 \mathrm{~S}$ ribosomal RNA by pyrosequencing and changes over time were compared.

59 sputum samples were collected during 24 pulmonary exacerbations from 23 subjects. Between treatment onset and day 3 there was a significant reduction in the relative abundance of Pseudomonas and increased microbial diversity. By day $8-10$, bacterial community composition was similar to pre-treatment. Changes in community composition did not predict improvements in lung function.

The relative abundance of Pseudomonas falls rapidly in subjects with CF receiving i.v. antibiotic treatment for a pulmonary exacerbation and is accompanied by an increase in overall microbial diversity. However, this effect is not maintained beyond the first week of treatment.

@ERSpublications

Changes in the CF microbiome in response to i.v. antibiotics are not sustained despite ongoing antibiotic pressure http://ow.ly/wzYyg

\footnotetext{
This article has supplementary material available from erj.ersjournals.com

Received: Nov 192013 | Accepted after revision: April 232014 | First published online: July 172014

Support statement: This work was supported by the Royal Hobart Hospital Research Foundation (Hobart, Australia) and the National Health and Medical Research Council of Australia (Canberra, Australia). D.J. Smith is the recipient of a Postgraduate scholarship from the National Health and Medical Research Council of Australia (APP1039309). G.J. Anderson is the recipient of a Senior Research Fellowship from the National Health and Medical Research Council of Australia (APP1024230). D.W. Reid is the recipient of a Practitioner Fellowship from the National Health and Medical Research Council of Australia. S.C. Bell and D.W. Reid are recipients of Queensland Health Medical Research Fellowships (QCOS013795 and QCOS013794).
}

Conflict of interest: Disclosures can be found alongside the online version of this article at erj.ersjournals.com

Copyright @ERS 2014 


\section{Introduction}

Despite advances in the management of cystic fibrosis (CF), chronic pulmonary infection remains responsible for most patient morbidity and mortality [1]. Culture-dependent analysis of CF airway infection reveals that Pseudomonas aeruginosa is the dominant bacterial pathogen in most adults with CF [2]. However, the recent application of culture-independent molecular techniques, based on the sequencing of the gene encoding bacterial 16S rRNA, have revealed a complex microbiome in the CF airway [3-9].

Chronic infection with $P$. aeruginosa is associated with an increased rate of lung function decline, increased frequency of pulmonary exacerbations, impaired quality of life and increased mortality [10, 11]. Guidelines recommend that pulmonary exacerbations are treated aggressively with a combination of at least two intravenous anti-pseudomonal antibiotics for 14 days [12, 13]. However, this practice is based on limited evidence [13].

Two studies, one prospective and one a large registry-based retrospective, have suggested that the maximum clinical and metabolic response to i.v. antibiotics occurs in the first week of treatment, with little additional benefit being achieved by extending treatment beyond this point $[14,15]$. Unfortunately, these studies did not assess the relationship between clinical improvement and microbiological response and their findings have not been considered sufficiently robust to change clinical practice.

In this study, we utilised culture-independent molecular techniques to explore the effect on the CF airway microbiome of i.v. antibiotic therapy administered for the treatment of an acute pulmonary exacerbation. The focus was primarily on the impact of antibiotic therapy on microbial community composition during the first week of treatment.

\section{Methods \\ Participants}

23 adult subjects (aged 18-54 years) with CF, admitted to hospital for i.v. antibiotic treatment of an acute, infective pulmonary exacerbation were recruited from The Prince Charles Hospital (Brisbane, Australia) $(n=14)$ and the Royal Hobart Hospital (Hobart, Australia) $(n=9)$. Institutional human research and ethics committee approval was obtained at both sites (HREC2008:2885 and H0009813, respectively). Individuals who had undergone lung transplantation or were using systemic immunosuppression were excluded. Based on standard microbiological cultures, all patients were infected with Pseudomonas aeruginosa (table S1).

Spontaneously expectorated sputum samples were collected at day 1 , before i.v. antibiotics (time-point (TP)-1), and at day 3-4 (TP-2) and day 8-10 (TP-3) following commencement of i.v. antibiotics.

A pulmonary exacerbation was defined clinically, based on the attending physician's assessment that the subject required i.v. antibiotics to treat an increase in respiratory symptoms or a decline in lung function.

Participant demographics, lung function and antibiotic treatment are outlined in table 1. Disease severity was determined based on the participants best forced expiratory volume in $1 \mathrm{~s}$ (FEV1) in the 12 months prior to recruitment ( $\mathrm{FEV} 1>70 \%$ mild, $40-70 \%$ moderate and $<40 \%$ severe).

\section{Sputum collection}

Sputum was expectorated directly into $10 \mathrm{~mL}$ of RNAlater (Life Technologies, Mulgrave, VIC, Australia) and stored for at least $24 \mathrm{~h}$ at $4{ }^{\circ} \mathrm{C}$ to allow full penetration into the sputum sample. Sputum samples were stored at $-80^{\circ} \mathrm{C}$ for later batch DNA extraction.

\section{DNA extraction}

A saliva-free aliquot of each frozen sputum sample was selected and manually homogenised with $500 \mu \mathrm{L}$ lysis buffer (50 mM Tris-HCl (Sigma-Aldrich, St Louis, MO, USA)), pH 6.8, $50 \mathrm{mM}$ ethylenediaminetetraacetic acid (AnalaR, PA, USA), $50 \mathrm{mM}$ sucrose (AnalaR), $100 \mathrm{mM}$ sodium chloride (Univar, Ingleburn, NSW, Australia) and $1 \%$ SDS (Amresco, Solon, OH, USA). The samples were incubated at $37^{\circ} \mathrm{C}$ for $1 \mathrm{~h}$ with $100 \mu \mathrm{L}$ chicken egg lysozyme $\left(100 \mathrm{mg} \cdot \mathrm{mL}^{-1}\right.$; Sigma-Aldrich), then at $56^{\circ} \mathrm{C}$ with shaking overnight with $100 \mu \mathrm{L}$ of proteinase $\mathrm{K}\left(20 \mathrm{mg} \cdot \mathrm{mL}^{-1}\right.$; Promega, Alexandria, NSW, Australia) and $75 \mu \mathrm{L}$ of $10 \%$ SDS. If samples were not completely digested, overnight incubation with additional proteinase was repeated until complete digestion was achieved. DNA was extracted and purified using PureLink Genomic DNA Mini Kit (Life Technologies) and eluted in $50 \mu \mathrm{L}$ kit elution buffer. Purified genomic DNA was analysed for quality $\left(\mathrm{A}_{260} / \mathrm{A}_{280}\right.$ ratio of 1.8-2.0) and concentration using a NanoDrop 1000 spectrophotometer (Thermo Fisher Scientific, Denver, CO, USA) and samples were diluted to $20 \mathrm{ng} \cdot \mu \mathrm{L}^{-1}$ prior to DNA amplification and sequencing. 
Real-time PCR quantification of P. aeruginosa

In 18 sputum samples from nine subjects, $P$. aeruginosa concentration was determined by real-time quantitative PCR using previously established methodologies [23]. Pearson's correlation was used to explore the relationship between $P$. aeruginosa load and relative abundance of Pseudomonas at the genus level.

\section{Results}

Sample quality

59 sputum samples were collected during 24 pulmonary exacerbations from 23 subjects. Sequencing yielded 420145 reads with an average read length of 457 base-pairs. After quality control and chimera detection, 57 samples (18 for TP-1, 18 for TP-2 and 21 for TP-3) and 336973 high-quality sequencing reads remained, with a median of 5478 sequences per sample and a range from 1468 to14 164 .

\section{Sputum microbiota before antibiotic treatment: TP-1}

18 sputum specimens were analysed at TP-1 and showed a complex microbiota, with a median (range) of 113 (23-210) OTUs per sample. A total of 100 different genera were observed (median (range) 13 (2-39) per sample) (fig. 1). Pseudomonas was the dominant genus in $94 \%$ (17 out of 18 ) of samples, with a mean (range) relative abundance of $78.5 \%$ (41.9-99.6\%). In one subject, Fusobacterium was the dominant genus (49\% of 16 S sequences), followed by Pseudomonas (41.9\% of sequences). Streptococcus was the second most abundant genus in 13 sputum samples, with a mean relative abundance of $7.3 \%$. The most prevalent anaerobic genera were Prevotella (mean (range) relative abundance 2.7\%, (0-13.8\%)) and Veillonella (mean (range) relative abundance $1.2 \%(0-5.9 \%)$ ), which were present in $72 \%$ and $78 \%$ of the subjects, respectively. Other pathogenic genera were recovered infrequently, including Actinomyces (44\% of samples), Staphylococcus (17\% of samples) and Haemophilus (11\% of samples). The mean percentage of sequences that could not be assigned to any known genus was 3\% (table S3). Statistical analysis of the taxonomic profiles at TP-1 did not identify a relationship between global microbial community composition (rank genus) and age (CCA: $\mathrm{p}=0.55$; ANOSIM: $\mathrm{p}=0.59$ ) or FEV1 (CCA: $\mathrm{p}=0.99$; ANOSIM: $\mathrm{p}=0.7$ ).

The relative abundance of Pseudomonas showed a strong negative correlation with microbial diversity (Shannon index; $\mathrm{r}=-0.81, \mathrm{p}<0.01$, Pearson correlation). Conversely, the relative abundance of Streptococcus $(\mathrm{r}=0.63, \mathrm{p}<0.01)$ and Prevotella $(\mathrm{r}=0.63, \mathrm{p}<0.01)$ were positively correlated with community diversity (fig. S1). We did not observe an association between microbial diversity and subject age $(\mathrm{p}=0.29)$ or lung function ( $\left.\mathrm{FEV}_{1} ; \mathrm{p}=0.3\right)$.

\section{Effects of antibiotic treatment on the airway microbiome}

There was a significant reduction in the relative abundance of Pseudomonas during the first $72 \mathrm{~h}$ of antibiotic treatment $(\mathrm{p}<0.005 ; \mathrm{FDR}=0.02$, paired t-test TP-1 versus TP- 2$)$. The mean relative abundance of Pseudomonas decreased from $78.5 \%$ of $16 \mathrm{~S}$ sequences before antibiotic treatment to $47 \%$ after $72 \mathrm{~h}$ of treatment. The reduction in Pseudomonas was accompanied by a significant increase in overall microbial diversity (Shannon index paired t-test $\mathrm{p}=0.012$ ) and a trend towards an increase in the relative abundance

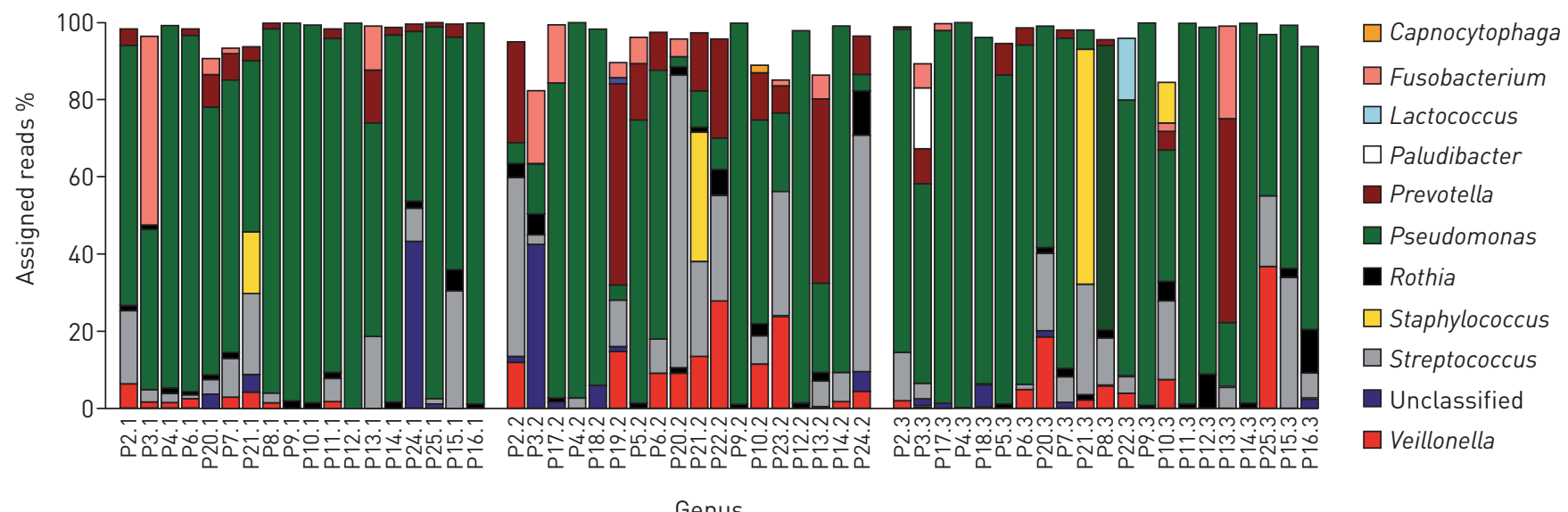

FIGURE 1 Taxonomic profile of all sputum samples segregated by time-point. Only genera which were present in at least one sample at a relative abundance of $\geqslant 10 \%$ are presented. 

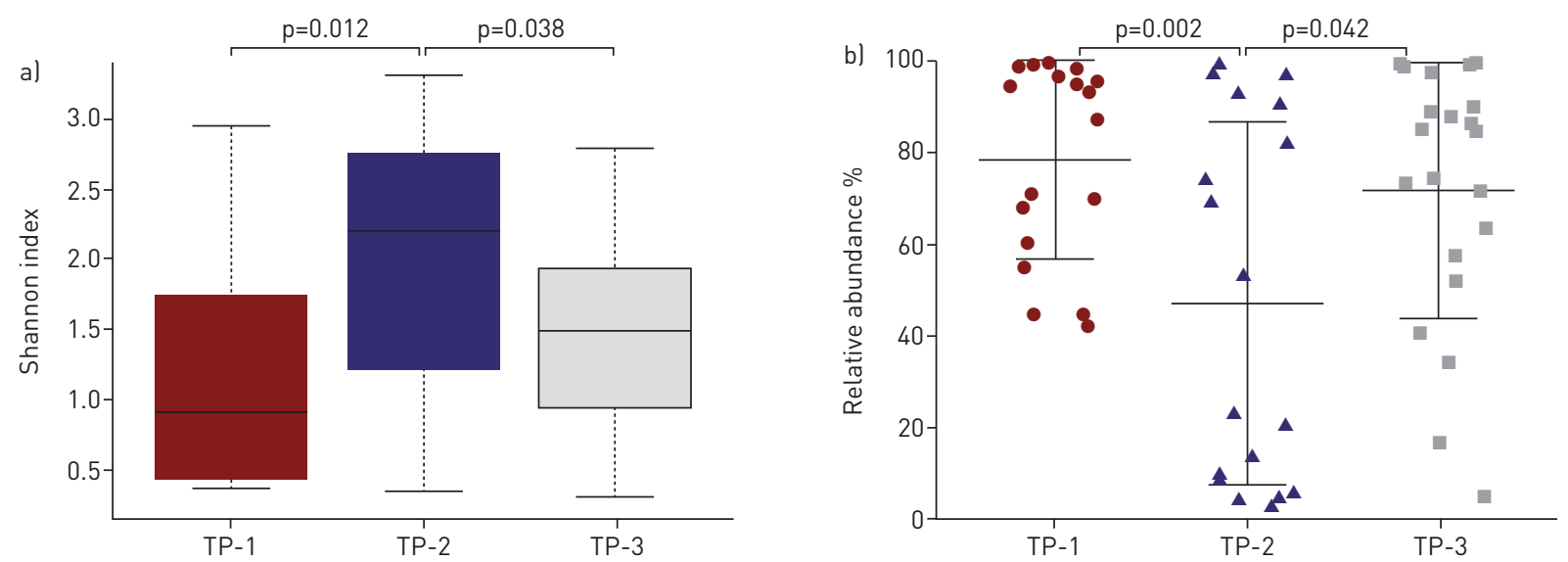

c)

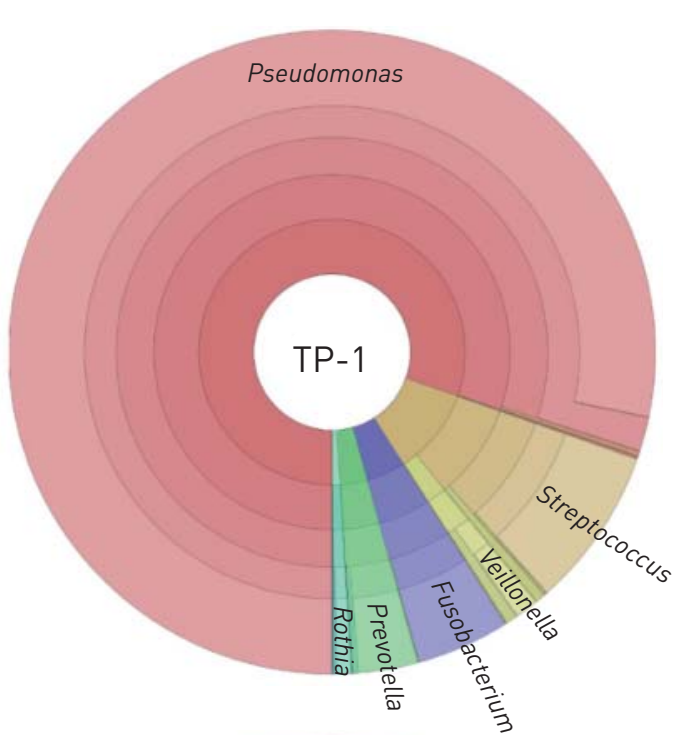

d)

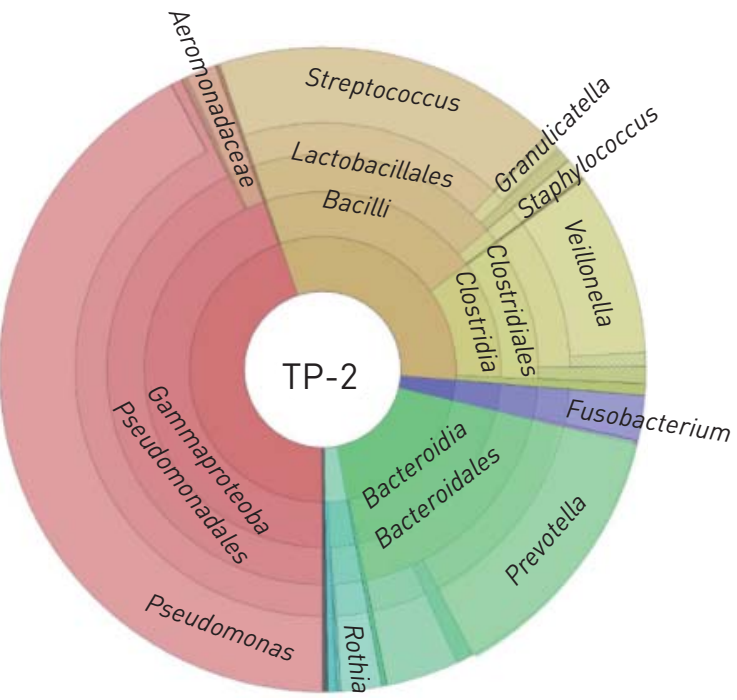

e)

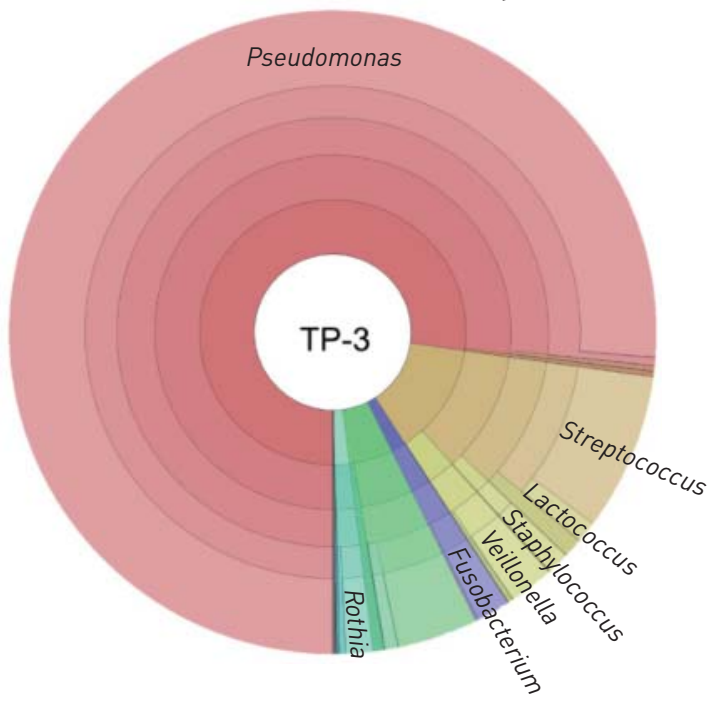

Proteobateria

Firmicutes

Fusobacteria

Bacteroidetes

Actinobacteria

Remaining

FIGURE 2 Changes in cystic fibrosis sputum microbiota between time-point (TP)-1, TP-2 and TP-3. a) Community diversity assessed by Shannon index (OTU level) demonstrating changes in overall microbial diversity. b) Changes in the relative abundance of Pseudomonas. c-e) Circle plots demonstrating changes in global community structure at c) TP-1, d) TP-2 and e) TP-3. Each ring represents a taxonomic rank (phylum, class, order, family and genus from inner to outer circle, respectively). 
of anaerobes, which was mainly driven by increases in the abundance of Prevotella (TP-1 2.7\% of $16 \mathrm{~S}$ sequences; TP-2 12.2\%; p=0.06; FDR=0.13) and Veillonella (TP-1 1.2\%; TP-2 6.8\%; p<0.02; FDR=0.07) (fig. 2).

At TP-3, overall microbial diversity (Shannon index) and Pseudomonas relative abundance were similar to TP-1, suggesting a return to pre-treatment community composition (fig. 2). The change in overall microbial diversity correlated negatively with the change in abundance of Pseudomonas $(\mathrm{r}=-0.7, \mathrm{p}<0.01)$ and positively with change in abundance of Streptococcus $(r=0.6, \mathrm{p}<0.05)$ (fig. S2).

A principal coordinates analysis demonstrated samples from TP-1 and TP-3 clustered together; however, TP-2 formed a distant separate cluster (fig. S3c). This clustering was significant; comparison of intra- and intergroup Jarrard distances of TP-1 versus TP-3 were not significant $(\mathrm{p}=0.75)$, but were significant between TP-1 versus TP-2 and TP-2 versus TP-3 $(\mathrm{p}<0.01)$.

We did not observe any significant associations between the changes in abundance of individual genera and improvement in FEV1 between TP-1 and TP-3. A negative relationship between relative abundance of Pseudomonas and FEV1 was observed at TP-3, but did not reach statistical significance $(\mathrm{r}=-0.46, \mathrm{p}=0.07)$.

In the nine subjects for whom $P$. aeruginosa quantification was performed, there was a weak, positive correlation between $P$. aeruginosa load and the relative abundance of Pseudomonas at the genus level $\left(\mathrm{r}^{2}=0.24, \mathrm{p}=0.04\right)$ (fig. S4).

\section{Sub-group analysis based on the relative abundance of $\mathrm{P}$. aeruginosa}

Clustering of OTUs based on the relative abundance of Pseudomonas spp. revealed three distinct groups: low abundance of Pseudomonas spp. $(<40 \%)$ with a complex community structure and presence of various other bacterial genera; medium abundance of Pseudomonas spp. (40 to $<75 \%$ ); and high abundance of Pseudomonas spp. ( $\geqslant 75 \%$ ) (fig. S3b).

Samples from subjects with severe disease tended to cluster in the high and medium categories, whereas samples from subjects with mild-to-moderate disease clustered in the low category (fig. S3c). Antibiotic treatment rarely resulted in a change in a subject's category at the end of treatment, and changes in the relative abundance of Pseudomonas in response to treatment were not associated with an improvement in lung function (fig. S2).

Non-Pseudomonas genera were categorised as obligate anaerobes or aerobes/facultative anaerobes (fig. 3). By TP-2 the median relative abundance of aerobic bacteria increased (TP-1: 4.5\% of 16S sequences; TP-2: $11.7 \%$ of $16 \mathrm{~S}$ sequences; $\mathrm{p}=0.03, \mathrm{FDR}=0.049)$ and a similar trend was observed in the abundance of anaerobic bacteria (TP-1: 3.4\%; TP-2: 22.6\%; $\mathrm{p}=0.1$ ). A reversal in these trends was seen between TP-2 and TP-3 (aerobes TP-3: median 9.5\%, paired t-test between TP-2 and TP-3 p =0.44; anaerobes TP-3: median: $2.4 \%$, $\mathrm{p}=0.048, \mathrm{FDR}=0.1$ ) and at TP-3, the sputum community composition once again resembled TP-1 (fig. 3 ).

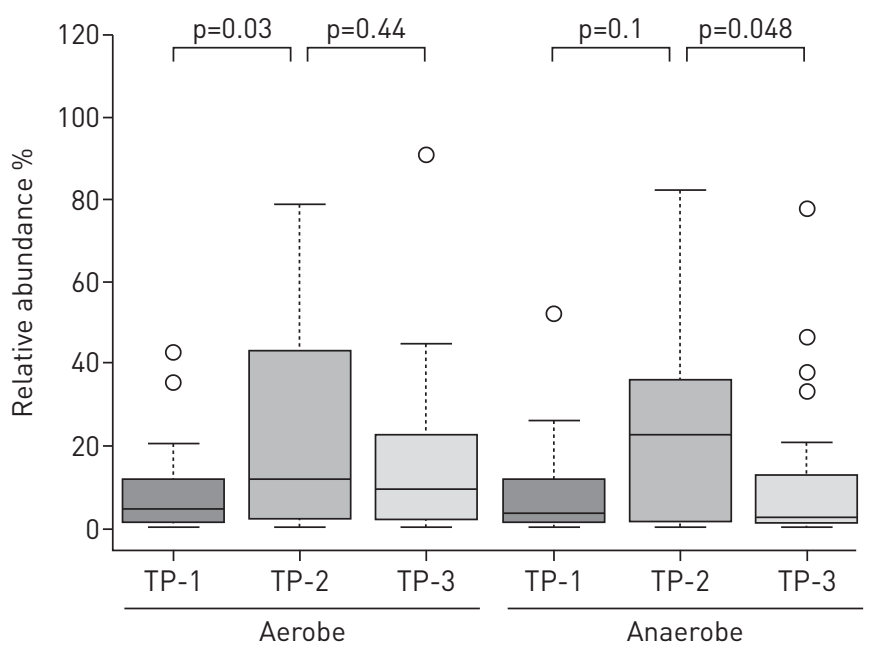

FIGURE 3 Relative abundance (median) of aerobic and anaerobic genera in cystic fibrosis sputum samples after exclusion of reads assigned to Pseudomonas. There was a significant increase in the relative abundance of aerobic bacteria between time-point (TP)-1 and TP-2, with a similar trend seen in anaerobic bacteria, followed by a significant decrease in anaerobic bacteria between TP-2 and TP-3. The p-values were calculated using a paired t-test. Changes in the relative abundance of aerobic and anaerobic genera in response to treatment in individual subjects are presented in figure S6. 


\section{Discussion}

In this study, we demonstrate a significant perturbation of the airway microbiome over the first $72 \mathrm{~h}$ of i.v. antibiotic treatment for pulmonary exacerbations in adult subjects with CF. This perturbation was characterised by a reduction in the dominance of Pseudomonas and an accompanying increase in microbial diversity. These changes were, however, short-lived, with bacterial community composition resembling that of the initial profile after 1 week of antibiotic treatment. These novel findings suggest that disturbance of the bacterial composition of the CF airway in response to i.v. antibiotics is transient, and potentially challenge current antibiotic management strategies for CF pulmonary exacerbations.

Evidence in the literature to support the application of culture-independent techniques to examine the effect of antibiotic therapy on the CF airway microbiome remains limited. TunNeY et al. [9] have demonstrated that despite total bacterial numbers being reduced in response to antibiotics (culture-based assessment), there was relative stability in overall community composition as assessed by terminal restriction fragment length polymorphisms analysis when sputum samples were collected from subjects with CF before and after treatment of a pulmonary exacerbation. More recently, DANIELS et al. [24] examined the impact of antibiotics on CF sputum microbial diversity in a group of adult CF subjects. Sputum samples collected following the initial $72 \mathrm{~h}$ of antibiotic therapy were compared with samples collected during a period of clinical stability prior to the exacerbation and after 10-14 days of treatment. In comparison to samples collected at $72 \mathrm{~h}$, samples collected at 10-14 days demonstrated an increase in relative abundance of Pseudomonas species compared with non-pseudomonads accompanied by a reduction in community diversity [24]. The authors concluded that antibiotic therapy was exerting a significantly greater effect on bacterial species other than Pseudomonas, culminating in the dominance of P. aeruginosa.

By examining microbial diversity at the onset of the exacerbation, prior to the administration of antibiotics, we have advanced the findings of DANIELs et al. [24] and shown that bacterial communities appear most susceptible to i.v. antibiotics at the beginning of antibiotic therapy. In addition to performing sampling prior to the onset of antibiotics, a number of important differences between the current study and that of DANIELS et al. [24] should be highlighted. In the earlier study, subjects were treated with a range of oral, inhaled and i.v. antibiotics which may have affected the ability to pick up consistent changes in community composition, whereas the subjects reported here were consistently treated with conventional i.v. antibiotic combinations. Additionally, sample processing and sequencing techniques differed between the two studies, most notably the samples in the study DANIELS et al. [24] were treated with propidium monoazide (PMA) to cross-link DNA from non-viable bacteria, a technique we did not employ. Nevertheless, both studies confirm that community composition remains essentially unchanged after at least 1 week of i.v. antibiotic therapy, which, given the early alterations in the microbiome that we observed, suggests that current treatment practices may need to be revised $[25,26]$.

The reduction in relative abundance of Pseudomonas from day 1 to days 3-4 suggests initial preferential killing of Pseudomonas in response to i.v. antibiotics. Recrudescence of $P$. aeruginosa infection under antibiotic pressure after this early response may occur as a result of increased replication of an inherently antibiotic resistant sub-population, or through population-wide adaptive mechanisms involving the upregulation of antibiotic resistance genes, which may occur rapidly in the treatment course [27, 28]. Whilst alternative explanations for the clinical improvements (reduction in respiratory symptoms and subjective wellbeing) in subjects beyond the first few days of treatment should also be considered, including the impact of adjunctive therapies, such as inhaled mucolytics, airway clearance techniques, rehydration and nutritional support $[14,29,30]$, our findings challenge the convention of prescribing the same i.v. antibiotic combination for more than a few days at a time. A potential new strategy may involve the rapid cycling of different antibiotic regimes during treatment, but this would require investigation in large-scale, adequately powered randomised and blinded clinical trials.

To explore the effect of antibiotics on bacterial species other than P. aeruginosa, we examined microbial community changes with Pseudomonas reads excluded. Contrary to the earlier study by DANIELs et al. [24] the relative abundance of both aerobes and anaerobes increased in the early stages of treatment, suggesting antibiotic therapy was having a lesser impact on the other bacterial species present compared to Pseudomonas. The effects of $i . v$. antibiotics in adults not infected with $P$. aeruginosa who are experiencing an acute exacerbation warrants further investigation, particularly as these individuals represent an increasing group of subjects transitioning from paediatric to adult care [31].

Greater sputum microbial diversity in our study subjects was positively associated with the abundance of Streptococcus, accompanied by a reduction in the relative abundance of Pseudomonas. These findings are consistent with a previous cross-sectional study in which microbial profiles of stable CF outpatients and inpatients were compared [32]. In this earlier report, three distinct sub-groups of CF subjects were identified by the relative abundance of Streptococcus and Pseudomonas in sputum. Importantly, outpatients 
with high Streptococcus and low Pseudomonas abundance had greater lung function stability over time. In our study, sputum samples with a high relative abundance of Pseudomonas and low community diversity formed a cluster, predominantly in subjects with severe lung disease. However, in contrast to other studies, we were unable to confirm a reduction in microbial diversity with increasing age and severity of lung disease in the CF subjects we studied $[25,33]$.

One potential limitation of our study is that PMA was not used to exclude DNA from non-viable bacterial cells in the sputum samples prior to analysis [34]. The use of PMA has been advocated due to appropriate theoretical concerns that molecular-based techniques may identify both viable and non-viable bacteria and limit the ability to detect changes in bacterial number in antibiotic treatment responses studies [35]. However, the reduction in the relative abundance of Pseudomonas we observed at day 3 would not be consistent with the inclusion of non-viable organisms [34]. The use of PMA remains a topic of debate and the method carries its own potential drawbacks, including a limited ability to expediently penetrate purulent, non-homogenised sputum prior to the death of resident organisms deep within the sputum sample. Furthermore, the extended processing time involved may result in less hardy bacteria (e.g. anaerobes) preferentially dying ex vivo, while more robust bacteria proliferate and skew the true bacterial composition, even if samples are maintained at $4^{\circ} \mathrm{C}[9,36]$.

Our conclusions are based on changes in relative abundance and not quantitative bacterial load. We have previously performed enumeration of $P$. aeruginosa number by real-time quantitative PCR and demonstrated heterogeneous changes in bacterial numbers in response to antibiotics [23]. The findings for some subjects in this earlier work were consistent with the current study, with an early reduction in $P$. aeruginosa numbers being followed by a recrudescence by the end of the first week of treatment [23]. Quantification of $P$. aeruginosa by real-time quantitative PCR in nine subjects from the current study revealed a positive correlation between $P$. aeruginosa load and the relative abundance of Pseudomonas at the genus level (fig. S4), and supports conjecture that changes in community composition in the CF lung reflect changes in $P$. aeruginosa concentration; however, this relationship was weak. Matching changes in community structure with enumeration of individual bacterial species is complex and changes in individual species may not be equivalent to changes at the level of the genus. Future advances in pyrosequencing technology may allow for identification and enumeration of bacteria at the species level, which will substantially advance understanding of the dynamics of the lung microbiome.

Approximately $3 \%$ of all obtained sequences in our study could not be assigned to a genus, which is consistent with the findings of other studies [37]. The success of read assignment is dependent on read length, primer pairs and the particular reference database applied to the analysis [38]. To date, there no universal method of analysis is agreed upon, which further confounds direct comparisons of the human microbiome between studies.

In summary, we have demonstrated for the first time that the relative abundance of $P$. aeruginosa falls rapidly in subjects with CF receiving i.v. antibiotics for pulmonary exacerbations, and that this is accompanied by an increase in microbial diversity. This effect was not maintained beyond the first week of treatment. These findings have implications for how i.v. antibiotic treatment should be employed for exacerbations and for how long. Future clinical trials should consider the impact of i.v. antibiotics on the whole lung microbiome in $\mathrm{CF}$, how changes in microbial community composition relate to reductions in absolute bacterial counts and, in turn, how these parameters relate to the clinical response to treatment.

\section{Acknowledgements}

We would like to thank to Dana Willner (Australian Centre for Ecogenomics, University of Queensland, Brisbane, Australia and Diamantina Institute, University of Queensland, Brisbane, Australia) for help and advice with the initial optimisation of DNA extraction protocols.

\section{References}

Cystic Fibrosis Foundation. Patient Registry: Annual Data Report 2010. Bethesda, MD, USA.

2 Williams HD, Davies JC. Basic science for the chest physician: Pseudomonas aeruginosa and the cystic fibrosis airway. Thorax 2012; 67: 465-467.

3 Rogers GB, Daniels TW, Tuck A, et al. Studying bacteria in respiratory specimens by using conventional and molecular microbiological approaches. BMC Pulm Med 2009; 9: 14.

4 de Vrankrijker AM, Wolfs TF, van der Ent CK. Challenging and emerging pathogens in cystic fibrosis. Paediatr Respir Rev 2010; 11: 246-254.

5 Harrison F. Microbial ecology of the cystic fibrosis lung. Microbiology 2007; 153: 917-923.

6 Sibley CD, Grinwis ME, Field TR, et al. Culture enriched molecular profiling of the cystic fibrosis airway microbiome. PLoS One 2011; 6: e22702.

7 Rogers GB, Carroll MP, Serisier DJ, et al. Characterization of bacterial community diversity in cystic fibrosis lung infections by use of $16 \mathrm{~s}$ ribosomal DNA terminal restriction fragment length polymorphism profiling. $J$ Clin Microbiol 2004; 42: 5176-5183. 
VanDevanter DR, LiPuma JJ. Microbial diversity in the cystic fibrosis airways: where is thy sting? Future Microbiol 2012; 7: 801-803.

9 Tunney MM, Klem ER, Fodor AA, et al. Use of culture and molecular analysis to determine the effect of antibiotic treatment on microbial community diversity and abundance during exacerbation in patients with cystic fibrosis. Thorax 2011; 66: 579-584.

10 Cohen-Cymberknoh M, Shoseyov D, Kerem E. Managing cystic fibrosis: strategies that increase life expectancy and improve quality of life. Am J Respir Crit Care Med 2011; 183: 1463-1471.

11 Kerem E, Viviani L, Zolin A, et al. Factors associated with FEV1 decline in cystic fibrosis: analysis of the data of the ECFS Patient Registry. Eur Respir J 2014; 43: 125-133.

12 Doring G, Flume P, Heijerman H, et al. Treatment of lung infection in patients with cystic fibrosis: current and future strategies. J Cyst Fibros 2012; 11: 461-479.

13 Plummer A, Wildman M. Duration of intravenous antibiotic therapy in people with cystic fibrosis. Cochrane Database Syst Rev 2011; 1: CD006682.

14 Bell SC, Bowerman AM, Nixon LE, et al. Metabolic and inflammatory responses to pulmonary exacerbation in adults with cystic fibrosis. Eur J Clin Invest 2000; 30: 553-559.

15 Collaco JM, Green DM, Cutting GR, et al. Location and duration of treatment of cystic fibrosis respiratory exacerbations do not affect outcomes. Am J Respir Crit Care Med 2010; 182: 1137-1143.

16 Dowd SE, Sun Y, Wolcott RD, et al. Bacterial tag-encoded FLX amplicon pyrosequencing (bTEFAP) for microbiome studies: bacterial diversity in the ileum of newly weaned Salmonella-infected pigs. Foodborne Pathog Dis 2008; 5: 459-472.

17 Caporaso JG, Kuczynski J, Stombaugh J, et al. QIIME allows analysis of high-throughput community sequencing data. Nat Methods 2010; 7: 335-336.

18 Haas BJ, Gevers D, Earl AM, et al. Chimeric 16S rRNA sequence formation and detection in Sanger and 454pyrosequenced PCR amplicons. Genome Res 2011; 21: 494-504.

19 Edgar RC. Search and clustering orders of magnitude faster than BLAST. Bioinformatics 2010; 26: 2460-2461.

20 DeSantis TZ, Hugenholtz P, Larsen N, et al. Greengenes, a chimera-checked 16S rRNA gene database and workbench compatible with ARB. Appl Environ Microbiol 2006; 72: 5069-5072.

21 Rogers GB, Cuthbertson L, Hoffman LR, et al. Reducing bias in bacterial community analysis of lower respiratory infections. ISME J 2013; 7: 697-706.

22 Ondov BD, Bergman NH, Phillippy AM. Interactive metagenomic visualization in a Web browser. BMC Bioinformatics 2011; 12: 385 .

23 Reid DW, Latham R, Lamont IL, et al. Molecular analysis of changes in Pseudomonas aeruginosa load during treatment of a pulmonary exacerbation in cystic fibrosis. J Cyst Fibros 2013; 12: 688-699.

24 Daniels TW, Rogers GB, Stressmann FA, et al. Impact of antibiotic treatment for pulmonary exacerbations on bacterial diversity in cystic fibrosis. J Cyst Fibros 2013; 12: 22-28.

25 Zhao J, Schloss PD, Kalikin LM, et al. Decade-long bacterial community dynamics in cystic fibrosis airways. Proc Natl Acad Sci USA 2012; 109: 5809-5814.

26 Stressmann FA, Rogers GB, Marsh P, et al. Does bacterial density in cystic fibrosis sputum increase prior to pulmonary exacerbation? J Cyst Fibros 2011; 10: 357-365.

27 Breidenstein EB, de la Fuente-Nunez C, Hancock RE. Pseudomonas aeruginosa: all roads lead to resistance. Trends Microbiol 2011; 19: 419-426.

28 Fothergill JL, Mowat E, Ledson MJ, et al. Fluctuations in phenotypes and genotypes within populations of Pseudomonas aeruginosa in the cystic fibrosis lung during pulmonary exacerbations. J Med Microbiol 2010; 59: 472-481.

29 Flume PA, Mogayzel PJ Jr, Robinson KA, et al. Cystic fibrosis pulmonary guidelines: treatment of pulmonary exacerbations. Am J Respir Crit Care Med 2009; 180: 802-808.

30 Flume PA, Robinson KA, O’Sullivan BP, et al. Cystic fibrosis pulmonary guidelines: airway clearance therapies. Respir Care 2009; 54: 522-537.

31 Lipuma JJ. The changing microbial epidemiology in cystic fibrosis. Clin Microbiol Rev 2010; 23 : $299-323$.

32 Filkins LM, Hampton TH, Gifford AH, et al. Prevalence of streptococci and increased polymicrobial diversity associated with cystic fibrosis patient stability. J Bacteriol 2012; 194: 4709-4717.

33 Cox MJ, Allgaier M, Taylor B, et al. Airway microbiota and pathogen abundance in age-stratified cystic fibrosis patients. PLoS One 2010; 5: e11044.

34 Rogers GB, Stressmann FA, Koller G, et al. Assessing the diagnostic importance of nonviable bacterial cells in respiratory infections. Diagn Microbiol Infect Dis 2008; 62: 133-141.

35 Rogers GB, Marsh P, Stressmann AF, et al. The exclusion of dead bacterial cells is essential for accurate molecular analysis of clinical samples. Clin Microbiol Infect 2010; 16: 1656-1658.

36 Tunney MM, Field TR, Moriarty TF, et al. Detection of anaerobic bacteria in high numbers in sputum from patients with cystic fibrosis. Am J Respir Crit Care Med 2008; 177: 995-1001.

37 Erb-Downward JR, Thompson DL, Han MK, et al. Analysis of the lung microbiome in the "healthy" smoker and in COPD. PLoS One 2011; 6: e16384.

38 Werner JJ, Koren O, Hugenholtz P, et al. Impact of training sets on classification of high-throughput bacterial 16s rRNA gene surveys. ISME J 2012; 6: 94-103. 